\title{
Working After Cancer. A Population-based Cohort Study Using A Life Course Perspective Approach. Régine Kiasuwa
}

R Kiasuwa ${ }^{1}$, V Nyaga ${ }^{1}$, C de Brouwer ${ }^{2}, C$ Bouland $^{2}$ ${ }^{1}$ Sciensano, Brussels, Belgium

${ }^{2}$ Université Libre de Bruxelles, Brussels, Belgium

Contact: regine.kiasuwambengi@sciensano.be

Introduction:

The prevalence of cancer survivors increases though in the workforce. The issue of their return-to-work represents an important matter for their quality of life, but also a great challenge. This study aims at quantifying the return-to-work of cancer survivors and at identifying the determining factors. Methods:

We requested data from the Belgian Cancer Registry and the Crossroad Bank for Social Security. We included socially insured Belgian workers diagnosed between 2004 and 2011 with colorectal, breast, head \& neck, prostate, testis, lung and corpus uteri cancer. The end of (administrative) follow-up was 31st December 2012.

After having solved legal, ethical and technical issues for the coupling, we performed group-based modeling for longitudinal data using the 'proc traj' package in SAS. We included demographic, health-related and work-related factors in the analysis and observed how these factors interplay to determine the working status.

Results:

Among the 38930 survivors included, after 5 years follow-up, $18 \%$ died, $69 \%$ were (partially) active, $31 \%$ were (pre-)retired, unemployed or disabled.

The group-based modeling revealed the existence of 3 types of work trajectories: high and stable; low and stable and changing across time. Different trajectories appear according to the follow-up time. Among the workers with high working probability, we mainly find women with breast cancer; men with prostate cancer and workers aged 36-50 years old.

Conclusions:

The results of our study reported that workers with cancer who resumed work were mainly self- employed and middle-age workers (36-50) with testis or breast cancers. Lung and prostate were negatively associated with the RTW and young workers seem to be at risk of unemployment.

It suggests that an early assessment of the worker's profile would allow the identification of those who would require support in order to prevent their exclusion from the labour market.

Key messages:

- The rate of cancer survivors who are active on the labour market five years after diagnosis is similar to the rate in the general population. 
154 European Journal of Public Health, Vol. 28, Supplement 4, 2018

- Age and profesisonal class are the main determining factors for the working probability after cancer. 